\title{
High Production of Small Organic Dicarboxylate Dianions by DESI and ESI
}

\author{
Yonghui Dong, ${ }^{1}$ Graziano Guella, ${ }^{2}$ Fulvio Mattivi, ${ }^{3}$ Pietro Franceschi ${ }^{1}$ \\ ${ }^{1}$ Biostatistics and Data Management, Research and Innovation Centre-Fondazione Edmund Mach, S. Michele all'Adige, TN, Italy \\ ${ }^{2}$ Bioorganic Chemistry Laboratory, Department of Physics, University of Trento, Povo, TN, Italy \\ ${ }^{3}$ Food Quality Research Group, Food Quality and Nutrition Department, Research and Innovation Centre-Fondazione Edmund \\ Mach, S. Michele all'Adige, TN, Italy
}

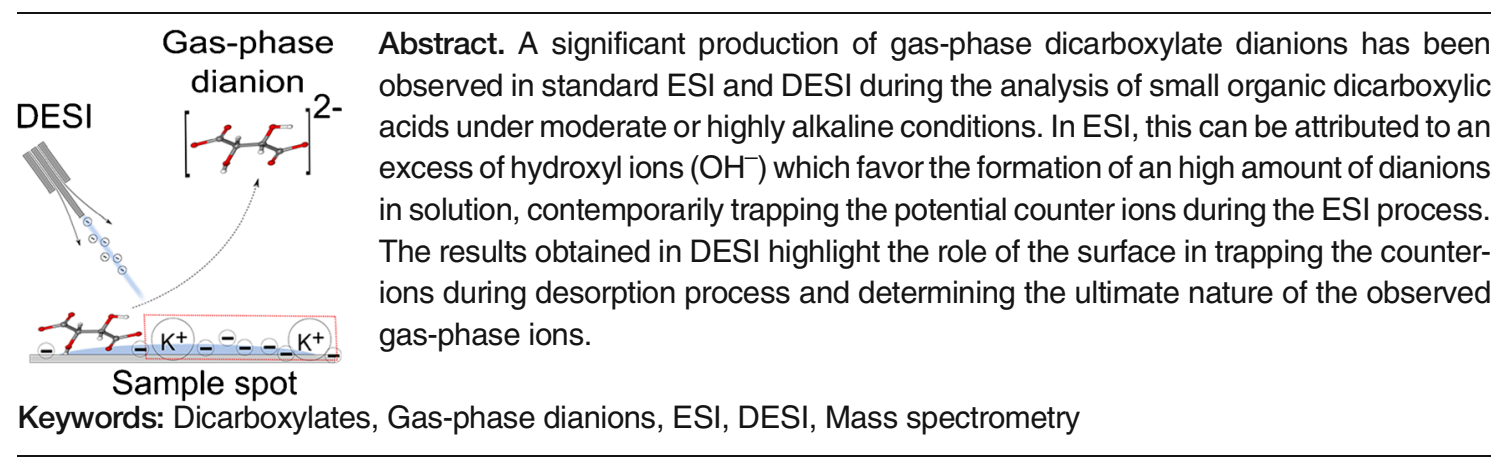

Received: 25 July 2014/Revised: 2 December 2014/Accepted: 10 December 2014/Published Online: 17 January 2015

\section{Introduction}

mall organic dicarboxylic acids play an important role in $\checkmark$ many biological systems. In the case of plants, they are key intermediates in carbon metabolism and may be present in high concentrations - often stored as $\mathrm{K}^{+}$salts [1]—with important implications for the production of beverages like juices or wine.

Since the $\mathrm{pKa}_{2}$ values in water of dicarboxylic acids $\left(\mathrm{H}_{2} \mathrm{DCA}\right)$, such as succinic, glutaric, adipic, malic, and tartaric acids, are lower than six $[2,3]$, in alkaline aqueous solutions they are expected to be present mostly as dicarboxylate dianions $\left(\mathrm{DCA}^{2-}\right)$, with relatively minor amounts of the monoprotonated $\mathrm{HDCA}^{-}$species. Despite this, small $\mathrm{DCA}^{2-}$ species are rarely (and barely) observed in the gas phase and it is speculated that this is due to the absence of the interactions with surrounding media (i.e., counterions or solvation). This type of interaction is always required to stabilize the charges [4-6]. From a general point of view, $\mathrm{DCA}^{2-}$ species can disappear through three main mechanisms: (1) protonation during solvent evaporation leading to $\mathrm{HDCA}^{-}$; (2) one electron

Electronic supplementary material The online version of this article (doi:10.1007/s13361-014-1065-y) contains supplementary material, which is available to authorized users.

Correspondence to: Pietro Franceschi; e-mail: pietro.franceschi@fmach.it loss leading, through an intermediate radical anion, to a decarboxylated singly charged radical anion $[7,8]$; or (3) dissociation into singly-charged negative ions.

For a given carbon backbone, the stability of $\mathrm{DCA}^{2-}$ in the gas phase can be increased by introducing additional functional groups, which allow charge delocalization and/or favor intramolecular hydrogen bonding, as in the case of tartaric acid [7]. This multifaceted behavior, coupled with a relatively simple structure, has made $\mathrm{H}_{2}$ DCA an ideal system for studying fundamental molecular phenomena like Coulomb repulsion [5] and solutesolvent interactions $[6,9]$. From an experimental point of view, ESI should be the favored technique for producing gas phase $\mathrm{DCA}^{2-}$, but it has always been quite difficult to produce these ionic species in significant amounts [10], at least for $\mathrm{H}_{2} \mathrm{DCA}$ with low molecular weight. In particular, a recent study by Tonner et al. [7] shows that tartaric dianions $\left(\mathrm{TA}^{2-}\right)$ can be produced by ESI, although not very efficiently, only under restricted experimental conditions, whereby their spontaneous thermodynamic decomposition via mechanism (b) is somewhat hindered. These calculations indeed indicate that $\mathrm{TA}^{2-}$ is metastable with respect to dissociation into the decarboxylated radical anion $+\mathrm{CO}_{2}+\mathrm{e}^{-}$.

Since it is widely accepted that desorption electrospray ionization (DESI) follows ESI-like dynamics [11-13], the production of $\mathrm{TA}^{2-}$ species in DESI-MS spectra was expected to 
be also highly inefficient. However, during DESI-MS imaging of $\mathrm{H}_{2} \mathrm{TA}$ in grape tissues (Figure 1), we observed a relatively strong signal at $\mathrm{m} / \mathrm{z} 74.00$ attributable to the tartrate dianion. This assignment was confirmed by high resolution measurements and isotope cluster analysis, which unambiguously assigned to this ion the $\mathrm{C}_{4} \mathrm{H}_{4} \mathrm{O}_{6}{ }^{2-}$ molecular formula. Therefore, $\mathrm{TA}^{2-}$ species could be produced in good yields under standard DESI-MS conditions. This outcome seemed to suggest the presence of significant and subtle differences between DESI and ESI for dianion production.

In order to investigate the $\mathrm{DCA}^{2-}$ formation mechanism, we designed a series of DESI and ESI experiments on $\mathrm{H}_{2} \mathrm{DCA}$ species of different carbon backbone lengths and with different numbers of hydroxyl groups. In particular, we analyzed: (a) five $\mathrm{H}_{2}$ DCA acids of general structural formula HOOC$\left(\mathrm{CH}_{2}\right)_{\mathrm{n}}$-COOH: succinic $\left(n=2, \mathrm{H}_{2} \mathrm{SA}\right)$, glutaric $(n=3$, $\left.\mathrm{H}_{2} \mathrm{GA}\right)$, adipic $\left(n=4, \mathrm{H}_{2} \mathrm{AA}\right)$, pimelic $\left(n=5, \mathrm{H}_{2} \mathrm{PA}\right)$, and suberic acids ( $\left.n=6, \mathrm{H}_{2} \mathrm{SuA}\right)$; (b) three hydroxylated dicarboxylic acids: tartronic (HOOC-CHOH-COOH, $\mathrm{H}_{2} \mathrm{TrA}$ ), malic (HOOC-CH2-CHOH-COOH, $\mathrm{H}_{2} \mathrm{MA}$ ), and L-(+)-tartaric acids (HOOC-CHOH-CHOH-COOH, $\mathrm{H}_{2} \mathrm{TA}$ ) (see Figure 2).

Since $\mathrm{H}_{2} \mathrm{DCA}$ species are often stored in grape tissue as potassium salts [1], different $\mathrm{H}_{2}$ DCA potassium salts - prepared by titration of the corresponding acid with $\mathrm{KOH}$ (potassium hydroxide) - were used as the starting point in all the MS experiments. In order to investigate the potential role played by counterions, the experiments were also performed on the corresponding sodium and lithium salts (for details see the Supporting Information). DESI analysis was carried out on $\mathrm{H}_{2} \mathrm{DCA}$ aqueous solutions deposited and air-dried on a chemically inert porous Teflon surface. For ESI measurements, the same $\mathrm{H}_{2}$ DCA aqueous solutions were directly injected into the ion source using a syringe pump. To minimize possible source-to-source differences, the source geometry of the DESI sprayer was adjusted to serve as the ESI source.

(a)
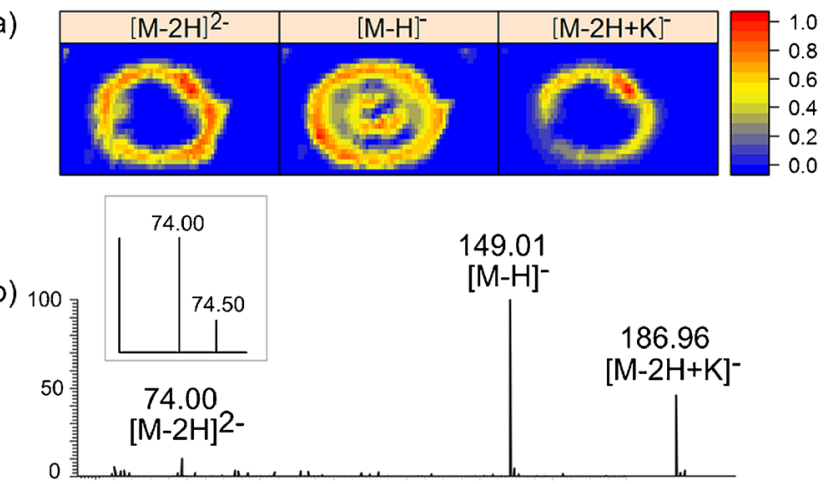

Figure 1. (a) DESI images of tartrate in grapevine leaf petiole. $[\mathrm{M}-2 \mathrm{H}]^{2-}$ denotes doubly charged tartrate dianions $\left(\mathrm{TA}^{2-}\right)$, [M$\mathrm{H}^{-}$singly deprotonated ion ( $\left.\mathrm{HTA}^{-}\right)$, and $[\mathrm{M}-2 \mathrm{H}+\mathrm{K}]^{-}$potassium adduct ions $\left(\mathrm{KTA}^{-}\right)$. (b) Representative spectrum of tartrate in grapevine leaf petiole. The insert shows the half-integer isotope spacing between the ions with molecular formulas ${ }^{12} \mathrm{C}_{4} \mathrm{H}_{4} \mathrm{O}_{6}{ }^{2-}$ at $m / z 74.00$ and ${ }^{12} \mathrm{C}_{3}{ }^{13} \mathrm{CH}_{4} \mathrm{O}_{6}{ }^{2-}$ at $\mathrm{m} / \mathrm{z} 74.50$

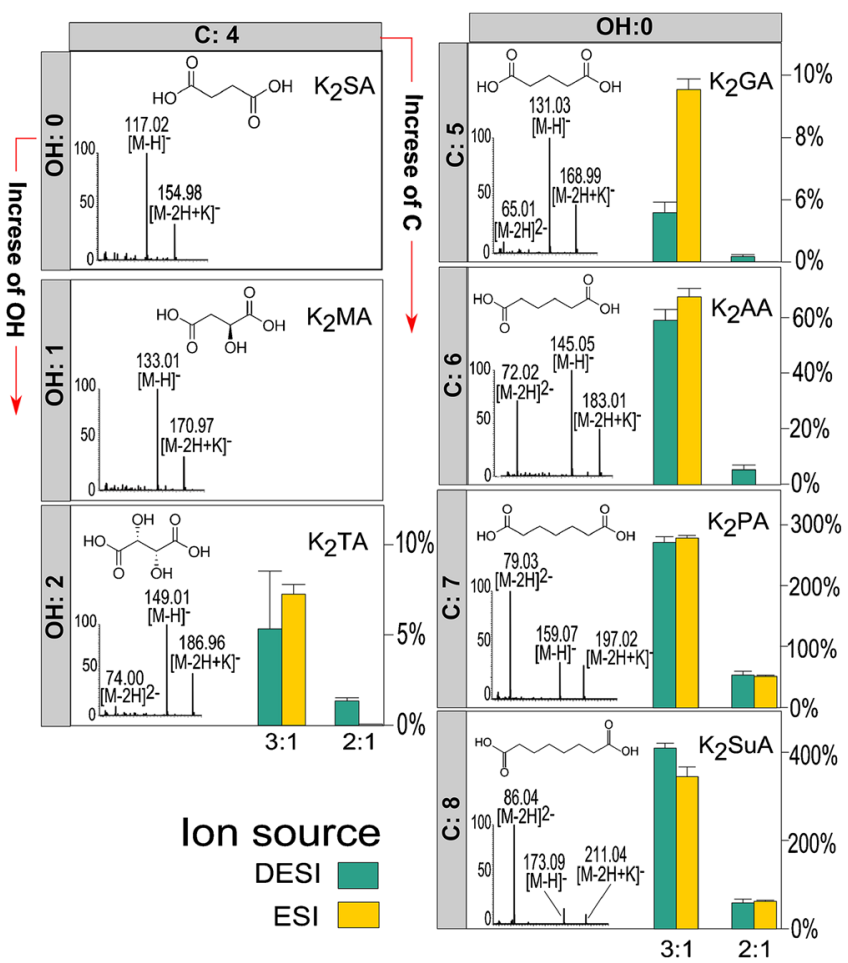

Figure 2. $\mathrm{DCA}^{2-}$ ion yields as observed in ESI and DESI ionsources for $\mathrm{K}_{2} \mathrm{DCA}$ aq. solutions. $\mathrm{DCA}^{2-}$ ion yields were calculated as the ratio of $[\mathrm{M}-2 \mathrm{H}]^{2-}$ abundances to those of their respective $[\mathrm{M}-\mathrm{H}]^{-}$singly-charged ions. Values represent mean $\pm \mathrm{SD}(n=6)$; ' $3: 1$ ' represents salts obtained from $\mathrm{H}_{2} \mathrm{DCA}$ using three molar equivalents $(3: 1)$ of $\mathrm{KOH}$, whereas ' $2: 1$ ' represents salts obtained from $\mathrm{H}_{2}$ DCA using two molar equivalents (2:1) of $\mathrm{KOH}$. The representative mass spectra shown in the plots are DESI spectra at ' $3: 1$ '. Results for $\mathrm{H}_{2}$ TrA are not shown here as no detectable signal for $\operatorname{TrA}^{2-}$ was found either in DESI or in ESI spectra

\section{Experimental}

Acetonitrile was purchased from Sigma-Aldrich (Milano, Italy), water was purified using a Mili-Q water purification system (Sartorius Stedim Biotech GmbH, Goettingen, Germany). Succinic $(>99.5 \%)$, malic $(>99.5 \%)$, glutaric $(>99.0 \%)$, and adipic acids $(>99.5 \%)$ were purchased from Fluka AG Buch, Switzerland; tartronic (97\%), L-(+)-tartaric $(99.5 \%)$, pimelic $(98 \%)$, suberic acids $(98 \%)$, lithium hydroxide monohydrate $(99 \%)$, sodium hydroxide $(>97 \%)$, and potassium hydroxide $(>85 \%)$ were from Sigma Aldrich.

The dicarboxylate salts were produced by mixing equal volumes $(20 \mathrm{~mL}$, water/acetonitrile 1:4) of $0.5 \mathrm{mM}$ aqueous solutions of each $\mathrm{H}_{2} \mathrm{DCA}$ (tartronic, succinic, malic, tartaric, glutaric, adipic, pimelic, and suberic acids) (a) with 3 molar equivalent $(1.5 \mathrm{mM})$ of alkali hydroxides $(\mathrm{LiOH}, \mathrm{NaOH}$, $\mathrm{KOH})$ or (b) with $2 \mathrm{~mol}$ equivalent $(1.0 \mathrm{mM})$ of the same hydroxides. Three $\mathrm{mL}$ of each final solution was deposited on a porous PTFE sheet (pore size $1-3 \mu \mathrm{m}$, porosity $25 \%-30 \%$, Porex Corporation, Bautzen, Germany) and then air dried 
before DESI analysis. Each solution was analyzed six times with an acquisition time of $1 \mathrm{~min}$.

All the MS experiments were performed using a ThermoFisher Scientific LTQ-Orbitrap XL mass spectrometer (Bremen, Germany) equipped with an OmniSpray ion source from Prosolia Inc. (Indianapolis, IN, USA). The typical instrumental parameters for standard DESI were: $-100 \mathrm{~V}$ tube lens voltage, $-10 \mathrm{~V}$ capillary voltage, $3 \mathrm{kV}$ spray voltage, $200^{\circ} \mathrm{C}$ capillary temperature, 2 micro scans, $200 \mathrm{~ms}$ MS injection time. Mass spectra were acquired in full scan negative mode over the $\mathrm{m} / \mathrm{z}$ range of 50250 . The DESI source parameters used were: $55^{\circ}$ spray angle, $1-$ $1.5 \mathrm{~mm}$ tip-to-surface distance, $\sim 4 \mathrm{~mm}$ tip-to-inlet distance, $10^{\circ}$ collection angle, 8 bar nitrogen carrier gas pressure. A mixture of Milli-Q water and acetonitrile (1:4, vol/vol) was used as spray solvent and delivered at the flow rate of $3 \mu \mathrm{L} \mathrm{min}^{-1}$.

ESI measurements were carried out by simply changing the DESI spray angle close to $45^{\circ}$, while keeping other instrumental and ion source parameters constant. Analyte solutions were injected directly into the inlet at the flow rate of $3 \mu \mathrm{L} \mathrm{min}^{-1}$. Each solution was analyzed six times with an acquisition time of $1 \mathrm{~min}$; ion source was washed after each analysis by spraying Milli-Q water/acetonitrile 1:4 (v/v) for about $10 \mathrm{~min}$ at the flow-rate of $8 \mu \mathrm{L} \mathrm{min}{ }^{-1}$.

\section{Results and Discussion}

The outcome of our complete set of experiments is summarized in Figure 2, which shows the intensity of the $\mathrm{DCA}^{2-}$ peak compared with those of their respective $[\mathrm{M}-\mathrm{H}]^{-}$singlycharged ions. Only the results for $\mathrm{K}_{2} \mathrm{DCA}$ salts are shown, but similar results were obtained for the corresponding sodium and lithium salts (see Supporting Information Figure S1 for $\mathrm{Na}_{2} \mathrm{DCA}$ and Figure $\mathrm{S} 2$ for $\mathrm{Li}_{2} \mathrm{DCA}$ salts).

Each panel of the plot displays the results relative to $\mathrm{H}_{2} \mathrm{DCA}$ species with different carbon backbones (second column) and for the $\mathrm{C} 4$ acids bearing different numbers of hydroxyl groups (first column). The bar plots illustrate the results obtained with aqueous solutions prepared from different $\mathrm{KOH} / \mathrm{H}_{2} \mathrm{DCA}$ ratios. Regardless of the ionization method or $\mathrm{KOH} / \mathrm{H}_{2} \mathrm{DCA}$ ratio, $\mathrm{DCA}^{2-}$ starts to be detectable for a carbon chain of at least five carbon atoms and, as expected, the relative intensity increases with the number of carbons. In ESI/DESI measurements carried out at different $\mathrm{KOH} / \mathrm{H}_{2} \mathrm{DCA}$ ratios on tartronic acid (HOOC-CHOH-COOH), the only 3-C dicarboxylic acid expected to give stable dianions, no detectable signal for the corresponding dianion was observed.

A longer chain means a greater separation between the two charges and the subsequent reduction of Coulomb repulsion stabilizes the doubly charged ion. Our findings are in keeping with previous investigations, which have shown that $\mathrm{DCA}^{2-}$ of HOOC- $\left(\mathrm{CH}_{2}\right)_{2}-\mathrm{COOH}$ is not stable in the gas phase $[6,14]$. A simple calculation of the Coulomb repulsion energy $E_{\text {rep }}=\mathrm{e}^{2} /$ $4 \pi \varepsilon_{0} \mathrm{r}$ between two electrons at distance $r$ suggests that the two negative charges in a molecule with a mutual electron affinity of $3 \mathrm{eV}$ (the expected value of carboxylate radical anions) may be stable when they are more than $5 \AA$ apart [7]. The charge separation distance of $5.3 \AA$ for succinate estimated by Wang and collaborators [5] explains why the bare succinate dianion is not observable in the gas phase. In our conditions, the increase in relative intensity with increased length of chain is remarkable: for HOOC- $\left(\mathrm{CH}_{2}\right)_{6}-\mathrm{COOH}$, the area of the $\mathrm{DCA}^{2-}$ species is significantly higher (four times) than that of the singly charged ion $\mathrm{HDCA}^{-}$. ESI and DESI seem to give qualitatively comparable results, although in ESI the relative yield was found to be slightly higher for glutaric acid $\left(\mathrm{H}_{2} \mathrm{GA}\right)$.

The effect of the hydroxyl groups on the four-carbon backbone is displayed in the first column of Figure 2. Only $\mathrm{H}_{2} \mathrm{TA}$ displays efficient production of dianions in both DESI and ESI. Again, this result is in agreement with the experiments and ab initio calculations reported recently [7], which showed that $\mathrm{TA}^{2-}$ is the smallest stable dicarboxylate in the gas phase. Interestingly, in our experiments we were able to obtain a much stronger signal for the $\mathrm{TA}^{2-}$ $\left(\mathrm{m} / \mathrm{z} 74\right.$, around $8 \%$ of $\left.\mathrm{HTA}^{-}\right)$than that reported in recent literature [7], where this species was barely detected, only through measurements carried out under highly restricted ESI conditions.

The strong yield observed here for the formation of $\mathrm{TA}^{2-}$ species is difficult to explain. We only note that ESI/DESI yield of this dianion is higher in strongly alkaline solutions as obtained by using a molar excess of metal-hydroxide (MOH). These conditions may give rise to two contrasting phenomena. On one hand, a strongly basic medium is required in order to favor the production of a high molar fraction of $\mathrm{TA}^{2-}$ species in the ESI/DESI conditions since the $\mathrm{pKa}_{1}$ and $\mathrm{pKa}_{2}$ of $\mathrm{H}_{2} \mathrm{TA}$ in acetonitrile/water are expected to be much higher than those in water. On the other hand, in a strongly basic medium the presence of higher concentration of the metal cation $\mathrm{M}^{+}$is expected to afford an overall depletion of $\mathrm{TA}^{2-}$ species. In fact, the increase of $\mathrm{M}^{+}$clearly promotes more efficient ion-pair aggregation leading to the formation of neutrals $\left(\mathrm{M}^{+}-\mathrm{TA}^{2-}-\mathrm{M}^{+}\right)$or singly charged species $\left(\mathrm{TA}^{2-}-\mathrm{M}^{+}\right)$during the ESI/DESI processes. The experimental results suggest that, in our conditions, the formation of these ion-pairs is not very efficient. As a possible interpretation of the observed phenomenon, we can hypothesize that during the transfer to the gas phase the metal ions can be selectively and efficiently "caged" by the stronger interaction with $\mathrm{OH}^{-}$in molar excess in the solution (ESI), making it possible for the $\mathrm{DCA}^{2-}$ to eventually reach the gas phase (Figure 3a).

To confirm the role of the free $\mathrm{OH}^{-}$in solution, we reduced its amount by using only two molar equivalent of $\mathrm{MOH}$ to prepare the initial solution. The results of the experiments under these conditions are summarized in the right bar plot in Figure 2 (2:1). The effect of reducing free $\mathrm{OH}^{-}$on the ESI spectra is striking: $\mathrm{DCA}^{2-}$ is practically undetectable in all ESI cases, at least for $\mathrm{H}_{2} \mathrm{DCA}$ species with low carbon atoms $(\mathrm{C} \leq$ 6). A minor signal is still visible $\left(0.075 \%\right.$ for $\mathrm{H}_{2} \mathrm{TA}, 0.006 \%$ for $\mathrm{H}_{2} \mathrm{AA}$ ), but it is akin to chemical noise and it is in line with what is reported in the literature [7]. Weak signals attributable to $\mathrm{PA}^{2-}$ and $\mathrm{SuA}^{2-}$ were nevertheless found in this condition, because of the longer distance between the two carboxylated groups of pimelic and suberic acids. This experiment unambiguously confirms the role of $\mathrm{OH}^{-}$for enhancing the yield of 


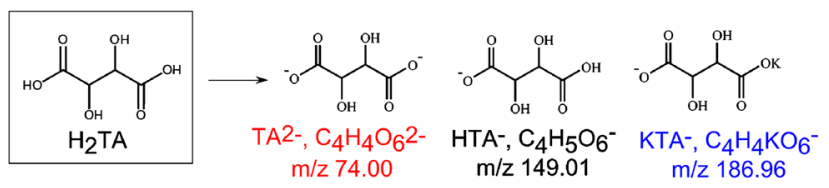

(a) ESI: KOH (3 molar equiv.)

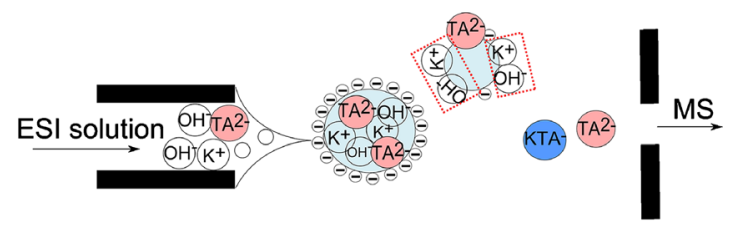

(b) DESI: KOH (2 molar equiv.)

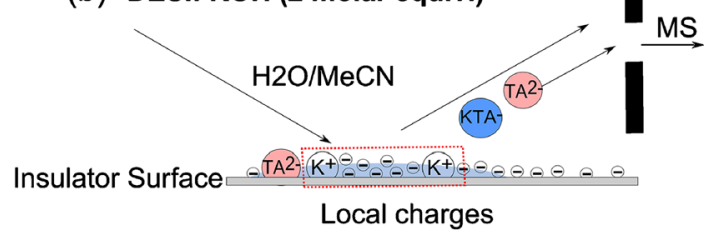

Figure 3. The proposed mechanisms of dianion production in $\mathrm{ESI}$ and DESI. In ESI, some of the $\mathrm{K}^{+}$ions are "caged" by the presence of one excess free $\mathrm{OH}^{-}$during ionization, and they are not available to completely neutralize the most abundant $\mathrm{DCA}^{2-}$ species in highly alkaline conditions. In DESI, even when there is no excess free $\mathrm{OH}^{-}$, the negative charges accumulated on the surface also "cage" $\mathrm{K}^{+}$ions during desorption, thereby favoring the release of free $\mathrm{DCA}^{2-}$ ions in the gas phase

dianions species, possibly by reducing the extent of ion pairing processes in ESI conditions.

In the DESI measurements on these $\mathrm{H}_{2}$ DCA 2:1 solutions the results, however, were different. Although the $\mathrm{DCA}^{2-}$ yield was lower than the one observed for $\mathrm{H}_{2} \mathrm{DCA}$ 3:1 solutions, the signals were still clearly present. A straightforward way to account for this observation is to suppose that in DESI there is another active "caging" mechanism that mimics the effects of the free $\mathrm{OH}^{-}$excess. The particular nature of DESI suggests a possible solution. It is known that DESI is a two-step process where the initial desorption of the analytes is followed by their ionization in an ESI-like process. The first step involves the landing and releasing of charges on the surface [15], and when the surface is an insulator, as in our case, this will produce a local charge accumulation [16]. In negative ion mode, the negatively charged surface can sequester the $\mathrm{K}^{+}$ions favoring the release of free $\mathrm{DCA}^{2-}$ ions in the gas phase (Figure $3 \mathrm{~b}$ ).

\section{Conclusions}

We have demonstrated a simple and efficient method for producing good yields of doubly charged dicarboxylates (in particular for small $\mathrm{H}_{2} \mathrm{DCA}$ such as tartaric, glutaric, and adipic acids) from their alkaline $\mathrm{H}_{2} \mathrm{O} / \mathrm{CH}_{3} \mathrm{CN}$ solutions under standard DESI and ESI conditions.

Our DESI results were obtained on a PTFE model surface, but the same phenomena are expected to occur on plant tissue sections, where the large amount of $\mathrm{OH}-$ rich polymers is expected to bind metal ions, favoring the formation of the $\mathrm{DCA}^{2-}$ observed during our imaging experiments. From an analytical point of view, our study suggests that $\mathrm{DCA}^{2-}$ signal could be used in DESI imaging experiments as a proxy to measure the distribution of $\mathrm{H}_{2}$ DCA salts in plant tissues. The potential application of this idea will be explored in further investigations.

\section{Acknowledgments}

The authors thank Dr. Ron Wehrens for helpful discussions.

\section{References}

1. Kliewer, V.M., Howarth, L., Omori, M.: Concentrations of tartaric acid and malic acids and their salts in Vitis vinifera grapes. Am. J. Enol. Vitic. 18, 42-54 (1967)

2. Gaber, A.A.A., Boraei, A.A., Abdalla, E.M.: Divalent transition metal ion mixed ligand complexes with aliphatic dicarboxylic acids and imidazoles. Transit. Met. Chem. 19, 435-438 (1994)

3. Harrington, C.F., Roberts, D.J., Nickless, G.: Optimization of a reversedphase high performance liquid chromatography separation using an ionpair reagent for the determination of carboxylic acids in plant materials. J. Liq. Chromatogr. Relat. Technol. 20, 1773-1787 (1997)

4. Wang, G.D., Colecor, R.B.: Effects of solvent and counterion on ion pairing and observed charge states of diquaternary ammonium salts in electrospray ionization mass spectrometry. J. Am. Soc. Mass Spectrom. 7, 1050-1058 (1996)

5. Wang, L.S., Ding, C.F., Wang, X.B., Nicolas, J.B.: Probing the potential barriers and intramolecular electrostatic interactions in free doubly charged anions. Phys. Rev. Lett. 81, 2667-2670 (1998)

6. Ding, C.F., Wang, X.B., Wang, L.S.: Photoelectron spectroscopy of doubly charged anions: intramolecular Coulomb repulsion and solvent stabilization. J. Phys. Chem. A 102, 8633-8636 (1998)

7. Tonner, R., Schwerdtfeger, P., May, A.L., Steill, J.D., Berden, G., Oomens, J., Campagna, S.R., Compton, R.N.: Stability of gas-phase tartaric acid anions investigated by quantum chemistry, mass spectrometry, and infrared spectroscopy. J. Phys. Chem. A 116, 4789-4800 (2012)

8. Kirk, B.B., Trevitt, A.J., Poad, B.L.J., Blanksby, S.J.: Characterisation of the ionic products arising from electron photodetachment of simple dicarboxylate dianions. Int. J. Mass Spectrom. 351, 81-94 (2013)

9. Yang, X., Fu, Y.J., Wang, X.B., Slavícek, P., Mucha, M., Jungwirth, P., Wang, L.S.: Solvent-mediated folding of a doubly charged anion. J. Am. Chem. Soc. 126, 876-883 (2004)

10. Wilfried, P.M.M., Nibbering, N.M.M.: Formation of doubly charged negative ions in the gas phase by collisionally-induced "ion pair" formation from singly charged negative ions. Int. J. Mass Spectrom. Ion Process. 88, 257-266 (1989)

11. Nefliu, M., Smith, J.N., Venter, A., Cooks, R.G.: Internal energy distributions in desorption electrospray ionization (DESI). J. Am. Soc. Mass Spectrom. 19, 420-427 (2008)

12. Takats, Z., Wiseman, J.M., Cooks, R.G.: Ambient mass spectrometry using desorption electrospray ionization (DESI): instrumentation, mechanisms, and applications in forensics, chemistry, and biology. J. Mass Spectrom. 40, 1261-1275 (2005)

13. Costa, A.B..., Cooks, R.G.: Simulated splashes: elucidating the mechanism of desorption electrospray ionization mass spectrometry. Chem. Phys. Lett. 464, 1-8 (2008)

14. Herbert, J.M., Ortiz, J.V.: Ab initio investigation of electron detachment in dicarboxylate dianions. J. Phys. Chem. A 104, 11786-11795 (2000)

15. Costa, A.B..., Cooks, R.G.: Simulation of atmospheric transport and droplet-thin film collisions in desorption electrospray ionization. Chem. Commun. 38, 3915-3917 (2007)

16. Benassi, M., Wu, C.P., Nefliu, M., Ifa, R.D., Volný, M., Cooks, R.G.: Redox transformations in desorption electrospray ionization. Int. J. Mass Spectrom. 280, 235-240 (2009) 